\title{
Características psicológicas relevantes para el rendimiento deportivo: diferencias de género en fútbol juvenil
}

\section{Psychological characteristics relevant to sports performance: gender differences in youth soccer}

\section{Características psicológicas relevantes para o desempenho esportivo: diferenças de gênero no futebol juvenil}

\author{
Olmedilla, A. ${ }^{1}$, Cánovas, M. ${ }^{1}$, Olmedilla-Caballero, B. ${ }^{1}$, Ortega, E. ${ }^{2}$ \\ ${ }^{1}$ Facultad de Psicología, CEIR Campus Mare Nostrum (CMN), Universidad de Murcia (Murcia, \\ España);
}

${ }^{2}$ Facultad de Ciencias del Deporte, CEIR Campus Mare Nostrum (CMN), Universidad de Murcia (San Javier, Murcia, España)

\begin{abstract}
RESUMEN
El estudio de los factores psicológicos relacionados con el rendimiento deportivo tiene un importante recorrido en la literatura científica. Conocer si existen diferencias de género en deportistas, cuáles son estas, y en qué aspectos se manifiestan es una cuestión que podría ayudar a mejorar la labor de entrenadores y técnicos deportivos. El objetivo de este estudio es determinar si existen diferencias de género en el perfil psicológico de futbolistas juveniles relacionado con el rendimiento deportivo. Se utilizó un diseño descriptivo transversal. La muestra objeto de estudio estuvo formada por un total de 329 jugadores juveniles de fútbol (46.8\% varones y $53,2 \%$ mujeres), con una edad media de $17.02( \pm 1.50)$ años. Para la evaluación de las características psicológicas se utilizó el Cuestionario Características Psicológicas Relacionadas con el Rendimiento Deportivo (CPRD). Los resultados de este estudio indican que existen diferencias estadísticamente significativas entre varones y mujeres en control de estrés (CE), influencia de la evaluación del rendimiento (IER) y en cohesión de equipo $(\mathrm{CH})$, con un tamaño del efecto de $.51, .31$ y .40 respectivamente. Los jugadores manifiestan puntuaciones mayores que las jugadoras en CE, es decir, gestionan mejor el estrés propio de la competición, y en IER, es decir, los jugadores gestionan mejor que las jugadoras el impacto que supone la evaluación de su rendimiento, el que hacen los otros (entrenadores, compañeros, padres/madres, etc.) y el que se hace a sí mismo. Sin embargo, las jugadoras presentan mejores puntuaciones en $\mathrm{CH}$, es decir, manifiestan una mayor tendencia a trabajar en grupo y fortalecer el equipo.
\end{abstract}

Palabras clave: Perfil psicológico, fútbol juvenil, rendimiento deportivo, diferencias de género, CPRD.

\section{ABSTRACT}

The study of psychological factors related to sports performance has an important track record in the scientific literature. Knowing if there are differences between male and female athletes, what they are, and 


\section{Olmedilla et al.}

in what aspects they manifest is an issue that could help improve the work of coaches and sports technicians. The aim of this study is to determine if there are gender differences in the psychological profile of youth soccer players related to sports performance. A descriptive cross-sectional design was used. The sample under study was formed by a total of 329 youth soccer players (46.8\% male and 53,2\% female), with an average age of $17.02( \pm 1.50)$ years. The Psychological Characteristics Questionnaire Related to Sports Performance (CPRD) was used to evaluate psychological characteristics. The results of this study indicate that there are statistically significant differences between male and female in stress control (CE), influence of performance evaluation (IER) and in team cohesion $(\mathrm{CH})$, with an effect size of $.51, .31$ and .40 respectively. The male players show higher scores than the female players in $\mathrm{CE}$, that is, they better manage the stress of the competition, and in IER, that is, the male players manage better than the female players the impact of evaluating their performance, the one that others do (coaches, partners, fathers / mothers, etc.) and the one that makes itself. However, female players have better $\mathrm{CH}$ scores, that is, they show a greater tendency to work in groups and strengthen the team.

Keywords: Psychological profile, youth soccer, sport performance, gender differences, CPRD.

\section{RESUMO}

O estudo de fatores psicológicos relacionados ao desempenho esportivo tem um histórico importante na literatura científica. Saber se existem diferenças entre atletas masculinos e femininos, o que são e em que aspectos eles se manifestam é uma questão que pode ajudar a melhorar o trabalho de treinadores e técnicos esportivos. O objetivo deste estudo é determinar se existem diferenças de gênero no perfil psicológico de jovens jogadores de futebol relacionados ao desempenho esportivo. Foi utilizado um desenho descritivo de corte transversal. A amostra estudada foi composta por um total de 329 jogadores de futebol juvenil (46,8\% masculino e 53,2\% feminino), com idade média de 17,02 $( \pm 1,50)$ anos. O Questionário de Características Psicológicas Relacionadas ao Desempenho Esportivo (CPRD) foi utilizado para avaliar as características psicológicas. Os resultados deste estudo indicam que existem diferenças estatisticamente significantes entre meninos e meninas no controle do estresse (EC), influência da avaliação de desempenho (IER) e na coesão da equipe (HC), com um tamanho de efeito de 0,51, 0,31 e 0,40, respetivamente. Os jogadores pequenos apresentam pontuações mais altas do que as jogadoras da CE, ou seja, administram melhor o estresse da competição, e no IER, ou seja, os jogadores pequenos gerenciam melhor do que as jogadoras o impacto de avaliar seu desempenho, o que os outros fazem (treinadores, parceiros, pais / mães, etc.) e o que se faz. No entanto, as jogadoras têm melhores pontuações no HC, ou seja, mostram maior tendência a trabalhar em grupo e fortalecer a equipe.

Palavras chave: Perfil psicológico, futebol juvenil, desempenho esportivo, diferenças de gênero, CPRD

\section{INTRODUCCIÓN}

El estudio de qué factores psicológicos se relacionan con el rendimiento deportivo tiene un importante recorrido y un gran desarrollo en la literatura científica (Olmedilla, García-Mas y Ortega, 2017; Orlick y Partington, 1988; Pastrana et al., 2019; Raglin, 2001; Reigal, Delgado, López y Hernández-Mendo, 2018; Williams y Krane, 1998), resultando ser, además, uno de los pilares fundamentales en los que se asientan los principios y metodologías del entrenamiento psicológico de los deportistas (Gould, Guinan, Greenleaf, Medbery y Peterson, 1999; Greenleaf, Gould y Dieffenbach, 2001), ya sean jóvenes en formación (Foster, Maynard, Butt y Hays, 2016), o profesionales y de élite (Einarsson, Kristjánsdóttir y Saavedra, 2019). Los resultados de esta línea de investigación indican una estrecha relación entre el rendimiento deportivo y los factores psicológicos, tanto en deportistas de élite como aficionados (Abdullah, Musa, Maliki, Kosni y Suppiah, 2016; Albuquerque, dos Santos, Greco y Da Costa, 2019; Pulido-Pedrero, de la Vega-Marcos y Fuentes-García, 2019; Swann et al., 2017). Además, una vez superada la perspectiva fenomenológica de la personalidad y su relación con el rendimiento de alto nivel (Olmedilla et al., 2018), criticada por los investigadores más orientados a la práctica, se pasó del estudio de los rasgos tradicionales al estudio de las estrategias 


\section{Diferencias psicológicas de género en fútbol juvenil}

mentales, habilidades y conductas que los deportistas utilizan para competir y su relación con el éxito deportivo (Vallarino, García y Zecchini, 2020; Weinberg y Gould, 2018), lo que favorece una perspectiva mucho más volcada en las aplicaciones prácticas de los hallazgos de la investigación. Así, se ha podido constatar que las características psicológicas resultan muy importantes por el impacto directo en el rendimiento del deportista (Bell, Knight, Lovett y Shearer, 2020; Fawver et al., 2020; Gillham y Stone, 2020; Hevilla-Merino y Castillo-Rodríguez, 2018), y por la mediación que desempeñan entre las habilidades físicas, técnicas y tácticas (Anderson, Hanrahan, y Mallett, 2014; Arthur, Fitzwater, Roberts, Hardy y Arthur, 2017; Mahamud, Tuero y Márquez, 2005).

El fútbol es un deporte de equipo de alta intensidad en el que se requieren habilidades físicas, técnicas, tácticas y psicológicas para un rendimiento óptimo (Gledhill, Harwood y Forsdyke, 2017; Olmedilla, Sánchez-Aldeguer, Almansa, Gómez-Espejo y Ortega, 2018; Stolen, Chamari, Castagna y Wisloff, 2005), y sobre el que se ha incrementado la investigación en los últimos años (Basevitch, 2019; Csáki, Szakály, Fózer-Selmec, Kiss y Bognár, 2017; Morris, 2000). Sin embargo, a pesar de que la irrupción de la mujer en el deporte de competición ha supuesto una tendencia creciente, no ha sido igual en todos los deportes, siendo el fútbol uno de ellos. En fútbol, la mayoría de estudios se han realizado con futbolistas varones, aunque empiezan a surgir investigaciones sobre fútbol femenino muy interesantes (Kristjánsdóttir, Jóhannsdóttir, Pic y Saavedra, 2019; Markovits y Hellerman, 2003; Olmedilla, Ruiz-Barquín, Ponseti, Robles-Palazón y García-Mas, 2019), aunque no hay muchos estudios que se centren en las diferencias entre varones y mujeres respecto a diferentes factores de rendimiento, incluido el psicológico (García-Naveira, Ruíz-Barquín y Ortín, 2015; Arias, Cardoso, Aguirre y Arenas, 2016). Conocer si existen diferencias entre varones y mujeres deportistas, cuáles son estas, y en qué aspectos se manifiestan es una cuestión que podría ayudar a mejorar la labor de entrenadores y técnicos deportivos. Así, evaluar las capacidades psicológicas es un primer paso para una correcta intervención psicológica y para mejorar el rendimiento del deportista, fundamental tanto para jugadores profesionales como para los futbolistas jóvenes en formación (Olmedilla et al., 2017).
Este estudio pretende determinar si existen diferencias de género en el perfil psicológico de futbolistas juveniles, concretamente respecto a su disposición psicológica relacionada con el rendimiento deportivo, es decir, control del estrés, evaluación del rendimiento, motivación, habilidades mentales y cohesión de equipo.

\section{MATERIAL Y MÉTODOS}

\section{Diseño}

Para el presente estudio se utilizó un diseño descriptivo transversal. Tal y como indican Ato, López-García y Benavente (2013) la finalidad en este tipo de diseños es explorar las relaciones entre variables con el objeto de pronosticar o explicar su comportamiento en un momento temporal específico determinado. Se caracterizan por tener una única muestra de participantes y porque los participantes son evaluados en un momento determinado sobre una o más variables.

\section{Participantes}

La muestra objeto de estudio estuvo formada por un total de 329 jugadores juveniles de fútbol, con una edad media de $17.02( \pm 1.50)$ años, y un rango de edad entre 15 y 19 años. Se realizó un muestreo de tipo intencional, teniendo en cuenta la proximidad física del club y la disponibilidad del mismo para participar en el estudio. Todos los jugadores pertenecían a clubes de fútbol de la Región de Murcia (España). El 46.8\% total de la muestra eran chicos y el 53,2\% eran chicas.

\section{Instrumentos}

Para la evaluación de las características psicológicas se utilizó el Cuestionario Características Psicológicas Relacionadas con el Rendimiento Deportivo (CPRD) de Gimeno, Buceta y Pérez-Llantada (2001). El CPRD es un cuestionario de auto-informe formado por 55 ítems de escala tipo Likert, con 5 alternativas de respuesta en un rango de 0 (totalmente en desacuerdo) a 4 (totalmente de acuerdo), y que se distribuyen en 5 factores (Control de Estrés - CE -; Influencia de la Evaluación del Rendimiento - IER -; Motivación MO -; Habilidad Mental - HM -; y Cohesión de Equipo - $\mathrm{CH}-$ ), con un alfa de Cronbach de 0.85, el cual se considera un coeficiente aceptable. A la hora de desglosar los factores, $\mathrm{CE}(0.88), \mathrm{CH}$ (0.78) e IER (0.72) se encuentran por encima de los valores 


\section{Olmedilla et al.}

mínimos aceptables de consistencia interna. En contraposición, los factores MO (0.67) y más concretamente el factor HM (0.34) se encuentra por debajo de los valores mínimos aceptados. Como aparece descrito en Gimeno et al. (2001) este cuestionario cuenta con los campos descriptivos necesarios para la notación de sexo, edad, número de años practicados, categoría, deporte y club.

\section{Procedimiento}

Para la realización de este estudio, lo primero que se hizo fue contactar con el director deportivo de la Federación de Fútbol de la Región de Murcia (FFRM). El director proporcionó el número de teléfono de los entrenadores y presidentes de los clubs en los que se iba a realizar el estudio. Se habló con los entrenadores y se quedó en una fecha y hora en el que se debía ir a pasar los cuestionarios. El protocolo de evaluación se explicó y se administró una media hora antes del horario de entrenamiento, generalmente en los vestuarios del campo de fútbol, y a todos los jugadores a la vez. Una vez explicado el objetivo de la investigación se pasó a explicar el CPRD haciendo hincapié en aquellos ítems en los que los sujetos pudieran tener alguna duda. El tiempo que se tardó en completar todos los instrumentos de evaluación osciló entre 20 y 25 minutos. En la última página se presentó un consentimiento informado, mediante el cual los sujetos aceptaban participar en el estudio voluntariamente. Con dicho consentimiento firmado, los mayores de 18 años podían irse a entrenar, mientras que a los menores se les entregó otra hoja de consentimiento que sus padres debían rellenar, firmar, y hacer llegar al investigador. Por otro lado, este estudio se realizó de acuerdo con las recomendaciones de la Declaración de Helsinki y fue aprobado por el Comité de Ética de la Universidad de Murcia (ID: UM 1551/2017).

\section{Análisis estadístico}

Se realizó un análisis descriptivo mediante medidas y desviaciones típicas. Para analizar las diferencias entre hombres y mujeres y tras la comprobación de la normalidad de los datos, se utilizó la prueba t de Student para muestras independientes. Se utilizó un p valor de .05. Para medir la magnitud del tamaño del efecto para las variables continuas, se utilizó la d de Cohen siguiéndose los rangos establecidos por Cohen (1988): < .000 (adverso), .000 - .199 (ningún efecto), .200 - .499 (pequeño), .500 - .799 (intermedio) y $.800-\geq 1.000$ (alto

Los datos fueron analizados en el programa informático SPSS 21.0.

\section{RESULTADOS}

En la Tabla 1 se pueden observar los valores medios y la desviación típica de las diferentes variables psicológicas objeto de estudio, según género. Los datos reflejan diferencias estadísticamente significativas entre chicos y chicas en $\mathrm{CE}$ (t327=4.595, p=.000), IER (t327=2.847, p=.005) y CH ( $\mathrm{t} 327=-3.517, \mathrm{p}=.000)$.

Tabla 1. Valores descriptivos y p valor de las sub-escalas del CRPD

\begin{tabular}{llllllllll}
\hline & \multicolumn{2}{c}{ Masculino } & \multicolumn{2}{c}{ Femenino } & \multicolumn{2}{c}{ Total } & & \\
& $M$ & $D T$ & $M$ & $D T$ & $M$ & $D T$ & $p$ & $d$ \\
\hline CE & 55.08 & 10.66 & 49.62 & 10.86 & 52.18 & 11.09 & .00 & .51 \\
\hline IER & 30.41 & 7.68 & 27.98 & 7.74 & 29.12 & 7.80 & .01 & .31 \\
\hline MO & 22.05 & 4.83 & 22.83 & 4.59 & 22.47 & 4.71 & .14 & .16 \\
\hline HM & 21.06 & 4.17 & 21.22 & 4.18 & 21.15 & 4.17 & .73 & .01 \\
\hline CH & 18.81 & 3.58 & 20.12 & 2.86 & 19.51 & 3.42 & .00 & .40 \\
\hline
\end{tabular}

Concretamente, los jugadores presentan puntuaciones mayores que las jugadoras en $\mathrm{CE}$, es decir, gestionan mejor el estrés propio de la competición, y en IER, es decir, los jugadores gestionan mejor que las jugadoras la evaluación que se hace de su rendimiento. Sin embargo, las jugadoras presentan mejores puntuaciones en $\mathrm{CH}$, es decir, manifiestan una tendencia mejor a trabajar en grupo y fortalecer el equipo.

En la Tabla 2 se muestran las puntuaciones centiles según los baremos establecidos por Gimeno et al. (2001). Se puede considerar que en ningún caso son puntuaciones altas (a mayor puntuación mejor disposición psicológica en cada uno de los factores), aunque siempre por encima del percentil 50 excepto $\mathrm{CH}$ en varones.

En la Figura 1 se aprecia el perfil de las cinco variables psicológicas en chicos y chicas, de manera que los 


\section{Diferencias psicológicas de género en fútbol juvenil}

primeros presentan valores más altos en CE e IER, y las chicas en MO, $\mathrm{HM} \mathrm{y} \mathrm{CH}$.

Tabla 2. Puntuaciones centiles en los baremos del CPRD de jugadores y jugadoras

\begin{tabular}{lccc}
\hline & Varones & Mujeres & Diferencia \\
\hline CE & 72 & 59 & $13-\mathrm{V}$ \\
\hline IER & 77 & 70 & $7-\mathrm{V}$ \\
\hline MO & 70 & 71 & $1-\mathrm{M}$ \\
\hline HM & 55 & 55 & $0-0$ \\
\hline CH & 45 & 65 & $20-\mathrm{M}$ \\
\hline
\end{tabular}

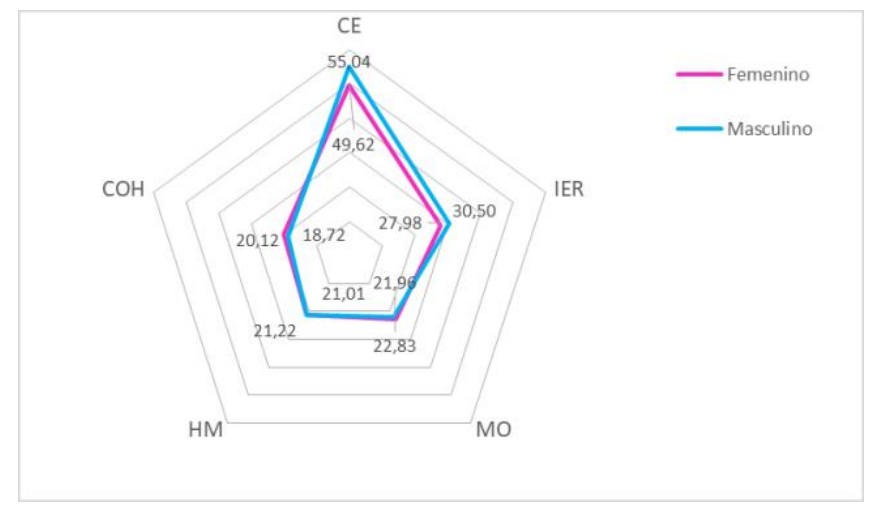

Figura 1. Perfil psicológico CPRD, según género

\section{DISCUSIÓN}

El objetivo de este estudio fue determinar si existen diferencias de género en el perfil psicológico de futbolistas juveniles, concretamente respecto a su disposición psicológica relacionada con el rendimiento deportivo, es decir, en aquellas características psicológicas relevantes para el rendimiento (CE, IER, MO, HM y $\mathrm{CH}$ ). Las características que evalúa el CPRD están en línea con lo señalado por otros autores que han identificado características psicológicas como la motivación, la autoconfianza, cognición autorreferencial, entre otras, que facilitaban el desarrollo de jóvenes deportistas con talento (Dohme, Piggott, Backhouse y Morgan, 2019; Höner y Feichtinger, 2016).
Los resultados de este estudio indican que existen diferencias estadísticamente significativas entre varones y mujeres en $\mathrm{CE}$, IER y $\mathrm{CH}$. Los jugadores presentan puntuaciones mayores que las jugadoras en $\mathrm{CE}$, es decir, gestionan mejor el estrés propio de la competición, y en IER, es decir, los jugadores gestionan mejor que las jugadoras el impacto que supone la evaluación de su rendimiento, el que hacen los otros (entrenadores, compañeros, padres/madres, etc.) y el que se hace a sí mismo. Sin embargo, las jugadoras presentan mejores puntuaciones en $\mathrm{CH}$, es decir, manifiestan una tendencia mejor a trabajar en grupo y fortalecer el equipo.

Las puntuaciones obtenidas por los jugadores y jugadoras en los dos factores relacionados con el estrés potencial de la práctica deportiva (CE y IER) indican que los primeros gestionan el estrés mejor que las segundas, que se ven menos afectados por situaciones potencialmente estresantes. Hallazgos en línea con lo encontrado en otros estudios, como el de Arias et al. (2016) donde los futbolistas (varones) también tienen puntuaciones superiores en IER a las futbolistas (mujeres), aunque en este caso no en CE. En este sentido, se puede considerar que la percepción de estrés en la práctica deportiva, ya sea en entrenamientos o en competiciones, parece ser vivida de manera diferente por los varones y por las mujeres. De hecho parece que las muejres tienden a valorar las situaciones estresantes como menos controlables (González y Sandoval, 2015; Hampel y Petermann, 2005). El fútbol ha sido históricamente un deporte practicado por hombres, con una carga de ideología masculina muy importante (Hargreaves, 1993; Uría, 2008), y la reciente incorporación de la mujer al mismo, puede provocar un sentido de responsabilidad en ésta que podría estar afectando su percepción de estrés. Las mujeres se enfrentan a unos estereotipos que se han generado durante muchos años y tienen mayor presión ante la sociedad que los hombres (Torrebadella-Flix, 2016). Por otro lado, es posible que por lo dicho anteriormente las jugadoras puedan tener una mayor exigencia a la hora de evaluar su propio rendimiento y una mayor sensibilidad a la evaluación que los demás puedan hacer sobre su rendimiento.

Por otro lado, considerando las puntuaciones centiles obtenidas se puede observar que, aunque los varones puntúan más alto que las mujeres en los factores de 


\section{Olmedilla et al.}

estrés, son puntuaciones medias-altas, es decir parece que afrontan adecuadamente las situaciones potencialmente estresantes, aunque queda un amplio margen de mejora. Este aspecto es mayor aún en las mujeres, y en ambos casos las puntuaciones en HM (similares en ambos grupos) indican que tanto jugadores como jugadoras no suelen utilizar estrategias y técnicas que incrementen sus destrezas y habilidades psicológicas. Estas puntuaciones bajas en HM coinciden con lo encontrado en otros estudios (López-López, 2011; Olmedilla et al., 2017). En el estudio de López-López (2011) los futbolistas aficionados presentaban puntuaciones significativamente menores en habilidades psicológicas que los futbolistas profesionales. En el estudio de Olmedilla et al. (2017) los jugadores de fútbol, pero también de rugby y baloncesto, muestran puntuaciones bajas en HM, lo que de alguna manera indica la necesidad de un trabajo específico de entrenamiento en destrezas psicológicas, interviniendo en variables como la motivación, concentración, cohesión de equipo, etc. con técnicas como la visualización, el establecimiento de objetivos, relajación a través de la respiración, entre otros (Aoyagi, Poczwardowski, Statler, Shapiro y Cohen, 2017; Brown y Fletcher, 2017; McCormick, Meijen, Anstiss y Jones, 2017). En este sentido, es importante tener en cuenta aquellos programas que ya han mostrado su eficacia en el entrenamiento de habilidades y estrategias psicológicas (MorenoFernández et al., 2019). Además, parece importante tener en cuenta las diferencias de género a la hora de abordar el entrenamiento psicológico (Einarsson et al., 2019), dado que tanto el repertorio de habilidades psicológicas (relajación, pensamiento negativo en la competición, diálogo interno durante la competición y visualización), como el interés hacia este tipo de trabajo no es igual para mujeres y varones. En el estudio de Einarsson et al. (2019) los varones utilizaron en mayor medida que las mujeres los programas de entrenamiento psicológico.

La evidencia empírica ha mostrado que el estrés es un factor que puede afectar tanto al rendimiento deportivo como a la salud, al bienestar y a la satisfacción por la práctica deportiva de los deportistas (Carolina-Paludo et al., 2020; Olmedilla, Ortega, Robles-Palazón, Salom y García-Mas, 2018). En este sentido, parece muy importante resaltar los hallazgos de este estudio en el sentido de que, si bien el aprendizaje de técnicas y estrategias psicológicas puede resultar fundamental tanto para las mujeres como los varones, en aquellas resulta mucho más necesario, lo que es coherente con diferentes estudios sobre el papel de los programas de entrenamiento psicológico (Olmedilla et al., 2019).

Por otro lado, y atendiendo las puntuaciones en $\mathrm{CH}$, los resultados de este estudio indican que las jugadoras presentan mejores puntuaciones que los jugadores, en línea con otros estudios (Arias et al., 2016). Es decir, las jugadoras manifiestan una mayor tendencia a trabajar en grupo y fortalecer el equipo, lo que es un dato muy importante desde el punto de vista de la cohesión y cooperación deportiva, y la importancia de ésta en el rendimiento y en el bienestar subjetivo percibido. De hecho, tal y como indica Basevitch (2019) las habilidades psicológicas necesarias para tomar decisiones óptimas y desempeñarse consistentemente en el fútbol de elite incluyen componentes individuales y de equipo, lo que determina el interés en las variables que influyen en la cohesión de equipo. Sin embargo, además de los aspectos positivos implicados desde la perspectiva de construcción de equipo, parece que también se puede relacionar el factor $\mathrm{CH}$ con la percepción de rendimiento individual, así en el estudio de Olmedilla et al. (2019), realizado con jugadoras de fútbol sub-16 y sub-18, el factor $\mathrm{CH}$ se relaciona negativamente con la percepción de rendimiento de la jugadora; quizá la exposición individual a la evaluación del rendimiento se viva con una elevada carga de estrés, y se "busque refugio" en la cohesión del equipo deportivo. Además, la percepción del propio rendimiento tiene mucho que ver con la influencia de la evaluación del rendimiento, aspecto estudiado en el factor IER del CPRD, en el que las jugadoras han mostrado puntuaciones menores que los jugadores.

Por último indicar que respecto al factor MO tanto los jugadores como las jugadoras presentan puntuaciones medio-altas, y similares, lo que en ambos casos indican que existe un margen importante de mejora; además es necesario tener en cuenta que este factor evalúa la motivación básica por el deporte practicado y la motivación cotidiana referida a las prácticas habituales de su deporte: entrenamientos, estilo de vida, viajes, compaginación estudios-deporte, etc.

Este estudio presenta algunas limitaciones que podrían y deberían ser consideradas en el futuro. Quizá la más 


\section{Diferencias psicológicas de género en fútbol juvenil}

relevante es el ser un estudio descriptivo, y aunque esto aporta información útil, sería interesante poder realizar estudios de carácter longitudinal lo que podría darnos información sobre la permanencia de determinados factores psicológicos, o el cambio de estos en función de programas específicos de trabajo, tal y como se han mostrado en otros estudios.

\section{CONCLUSIONES}

Los futbolistas juveniles chicos respecto a las chicas tienen puntuaciones más altas en control de estrés y en influencia de la evaluación del rendimiento, lo que indica que controlan mejor el estrés propio de la competición y que gestionan mejor la evaluación que se hace de su rendimiento.

Las futbolistas juveniles chicas respecto a los chicos tienen puntuaciones más altas en cohesión de equipo, lo que indica que se sienten más atraídas e identificadas con el grupo deportivo, tanto en referencia a relaciones informales, como en la cohesión relativa a relaciones estrictamente deportivas.

\section{APLICACIONES PRÁCTICAS}

Los resultados de este estudio permiten conocer algunas de las características psicológicas más importantes en relación al rendimiento deportivo tanto para los jugadores como para las jugadoras de fútbol juvenil. Como se ha visto existen diferencias que son necesarias tener en cuenta a la hora de implementar acciones para la planificación de entrenamientos y preparación de competiciones. Tal y como indican Bell et al. (2020) el informar a los deportistas desde jóvenes de los beneficios de la psicología del deporte, el mostrarles la variedad de características psicológicas que poseen los deportistas de élite y las habilidades psicológicas disponibles que se usan normalmente para mejorar el rendimiento, ayudaría a la implementación de programas. Otro aspecto a destacar podría ser educar y formar a los entrenadores sobre el tema de la psicología del deporte dada su influencia en los deportistas, por ejemplo conociendo la influencia que las propuestas de juegos tácticos en los entrenamientos tienen en la carga mental del jugador (Cárdenas, Conde-González y Perales, 2015; García-Calvo, González-Ponce, Ponce, ToméLourido y Vales-Vázquez, 2019), o los factores para la construcción de la identidad competitiva en edades tempranas (Merino, Arraiz y Sabirón, 2019). En esta línea, si por un lado se puede contar con la aportación de psicólogos o psicólogas del deporte estos deberán, en función de estos datos, implementar programas específicos de trabajo psicológico, y ayudar tanto a jugadores y jugadoras como a entrenadores y técnicos a abordar adecuadamente un trabajo interdisciplinar para el entrenamiento y la preparación de las competiciones.

\section{AGRADECIMIENTOS}

Agradecer a la Federación de Fútbol de la Región de Murcia (FFRM) todo su apoyo y disponibilidad para la realización de este estudio Asimismo agradecer a los clubes, entrenadores y técnicos participantes su disposición, y sobre todo a los y las futbolistas por su participación generosa e implicada.

Financiación: Este estudio fue parcialmente financiado por la Federación de Fútbol de la Región de Murcia (FFRM), España, dentro del Proyecto de Investigación con número de registro FFRM-UMU-04 0092 321B 6450214704.

\section{REFERENCIAS}

1. Abdullah, M. R., Musa, R. M., Maliki, A. B. H. M., Kosni, N. A. y Suppiah, P. K. (2016). Role of psychological factors on the performance of elite soccer players. Journal of Physical Education and Sport, 16(1), 170-176. DOI:10.7752/jpes.2016.01027

2. Albuquerque, M. R., dos Santos, A., Greco, P. J. y Da Costa, I. T. (2019). Asociación entre el control inhibitorio y el rendimiento táctico de los jugadores de fútbol sub-15. Revista de Psicología del Deporte, 28(1), 63-70. https://doi.org/10.18002/10612/910

3. Anderson, R., Hanrahan, S. J. y Mallett, C. J. (2014). Investigating the optimal psychological state for peak performance in Australian elite athletes. Journal of Applied Sport Psychology, 26, 318-333. doi: 10.1080/10413200.2014.885915.

4. Aoyagi, M. W., Poczwardowski, A., Statler, T., Shapiro, J. L. y Cohen, A. B. (2017). The performance interview guide: 


\section{Olmedilla et al.}

recommendations for initial consultations in sport and performance psychology. Professional Psychology: Research and Practice 48, 352360. https://doi.org/10.1037/pro0000121

5. Arias, I., Cardoso, T., Aguirre, H. y Arenas, J. (2016). Características psicológicas de rendimiento deportivo en deportes de conjunto. Psicogente, 19(35), 25-36. http://doi.org/10.17081/psico.19.35.1206

6. Arthur, R. A., Fitzwater, J., Roberts, R., Hardy, J. y Arthur, C. A. (2017). Psychological skills and "the paras": the indirect effects of psychological skills on endurance. Journal of Applied Sport Psychology, 29, 449-465. doi: 10.1080/10413200.2017.1306728

7. Ato, M., López-García, J. J. y Benavente, A. (2013). Un sistema de clasificación de los diseños de investigación en psicología. Anales de Psicología/Annals of Psychology, 29(3), 1038-1059.

https://doi.org/10.6018/analesps.29.3.178511

8. Basevitch, I. (2019). Psychological Demands of Soccer: A Decision-Making Perspective. In R. Curtis, C. Benjamin, R. Huggins y D. Casa (Eds.), Elite Soccer Players (pp. 19-34). Routledge: Taylor \& Francis, UK.

9. Bell, A. F., Knight, C. J., Lovett, V. E. y Shearer, C. (2020). Understanding Elite Youth Athletes' Knowledge and Perceptions of Sport Psychology. Journal of Applied Sport Psychology, 10.1080/10413200.2020.1719556.

10. Brown, D. J. y Fletcher, D. (2017). Effects of psychological and psychosocial interventions on sport performance: a meta-analysis. Sports Medicine, 47, 77-99. https://doi.org/10.1007/s40279-016-0552-7

11. Cárdenas, D., Conde-González, J. y Perales, J. C. (2015). El papel de la carga mental en la planificación del entrenamiento deportivo. Revista de Psicología del Deporte, 24(1), 91-100.

https://doi.org/10.1016/j.ramd.2016.04.001

12. Carolina-Paludo, A., Nunes-Rabelo, F., Maciel-Batista, M., Rúbila-Maciel, I., Peikriszwili-Tartaruga, M. y Simões, A. C. (2020). Game location effect on pre-competition cortisol concentration and anxiety state: A case study in a futsal team. Revista de Psicología del Deporte, 29(1), 105-112.

13. Cohen, J. (1988). Statistical power analysis for the behavioral sciences (2a Ed.). Hillsdale, NJ: Erlbaum.

14. Csáki, I., Szakály, Z., Fózer-Selmec, B., Kiss, S. Z. y Bognár, J. (2017). Psychological and anthropometric characteristics of a Hungarian elite football academy's players. Physical Culture and Sport. Studies and Research, 73(1), 15-26. DOI: 10.1515/pcssr2017-0002.

15. Dohme, L. C., Piggott, D., Backhouse, S. y Morgan, G. (2019). Psychological skills and characteristics facilitative of youth athletes' development: A systematic review. The Sport Psychologist, $\quad 33(4), \quad$ 261-275. https://doi.org/10.1123/tsp.2018-0014.

16. Einarsson, E. I., KristjánsdÓttir, H. y Saavedra, J. M. (2019). Relationship between elite athletes' psychological skills and their training in those skills. Nordic Psychology, 1-10. https://doi.org/10.1080/19012276.2019.162999 $\underline{2}$.

17. Fawver, B., Cowan, R. L., DeCouto, B. S., Lohse, K. R., Podlog, L. y Williams, A. M. (2020). Psychological characteristics, sport engagement, and performance in alpine skiers. Psychology of Sport and Exercise, 47, 101616. https://doi.org/10.1016/j.psychsport.2019.10161 6.

18. Foster, D., Maynard, I., Butt, J. y Hays, K. (2016). Delivery of psychological skills training to youngsters. Journal of Applied Sport Psychology, 28(1), 62-77. https://doi.org/10.1080/10413200.2015.106309 7.

19. García-Calvo, T., González-Ponce, I., Ponce, J. C., Tomé-Lourido, D. y ValesVázquez, Á. (2019). Incidencia del sistema de puntuación de las tareas sobre la carga mental del entrenamiento en fútbol. Revista de Psicología del Deporte, 28(2), 79-86. https://doi.org/10.6018/sportk.431161 


\section{Diferencias psicológicas de género en fútbol juvenil}

20. García-Naveira, A., Ruiz-Barquín, R. y Ortín, F. J. (2015). Optimismo y competitividad en jóvenes atletas de rendimiento. Revista Latinoamericana de Psicología, 47(2), 124-135. https://doi.org/10.1016/j.rlp.2014.08.001.

21. Gillham, A. y Stone, C. (2020). Experiences of Peak Performance in Elite American Football. The Sport Psychologist, 1(aop), 1-12. https://doi.org/10.1123/tsp.20180132.

22. Gimeno, F., Buceta, J. M. y PérezLlantada, M. (2001). El cuestionario "Características psicológicas relacionadas con el rendimiento deportivo" (CPRD): Características psicométricas. Análise Psicológica, 1(19), 93133. https://doi.org/10.14417/ap.346

23. Gledhill, A., Harwood, C. y Forsdyke, D. (2017). Psychosocial factors associated with talent development in football: A systematic review. Psychology of Sport and Exercise, 31, 93-112.

https://doi.org/10.1016/j.psychsport.2017.04.00 2.

24. González, J. y Sandoval, J. M. (2015). Estrategias de afrontamiento y personalidad en la adaptación de jóvenes a su práctica deportiva. Diferencias de género. SPORT TK-Revista EuroAmericana de Ciencias del Deporte, 4(1), 57-62. DOI: https://doi.org/10.6018/239831.

25. Gould, D., Guinan, D., Greenleaf, C., Medbery, R. y Peterson, K. (1999). Factors affecting Olympic performance: Perceptions of athletes and coaches from more and less successful teams. The Sport Psychologist, 13(4), 371-394.

DOI: https://doi.org/10.1123/tsp.13.4.371.

26. Greenleaf, C., Gould, D. y Dieffenbach, K. (2001). Factors influencing Olympic performance: interviews with Atlanta and Negano US Olympians. Journal of Applied Sport Psychology, 13(2), 154-184. https://doi.org/10.1080/104132001753149874.

27. Hampel, P. y Petermann, F. (2005). Age and Gender Effects on Coping in Children and Adolescents. Journal of Youth and Adolescence,
34, 2, 73-83. https://doi.org/10.1007/s10964005-3207-9.

28. Hargreaves, J. (1993). Promesa y problemas en el ocio y los deportes femeninos. En J. I. Barbero (comp.), Materiales de sociología del deporte (pp. 109-132). Madrid: La Piqueta.

29. Hevilla-Merino, A. y Castillo-Rodríguez, A. (2018). Fluctuación de las características psicológicas de rendimiento deportivo en jóvenes futbolistas. Estado basal vs. Precompetitivo. Cuadernos de Psicología del Deporte, 18(3), 169-178. https://doi.org/10.4321/s1578-

$\underline{84232013000200003}$

30. Höner, O. y Feichtinger, P. (2016). Psychological talent predictors in early adolescence and their empirical relationship with current and future performance in soccer. Psychology of Sport and Exercise, 25, 17-26. https://doi.org/10.1016/j.psychsport.2016.03.00 $\underline{4}$.

31. Kristjánsdóttir, H., Jóhannsdóttir, K. R., Pic, M. y Saavedra, J. M. (2019). Psychological characteristics in women football players: Skills, mental toughness, and anxiety. Scandinavian Journal of Psychology, 60(6), 609-615. https://doi.org/10.1111/sjop.12571.

32. López-López, I. S. (2011). La evaluación de variables psicológicas relacionadas con el rendimiento en fútbol: habilidades psicológicas para competir y personalidad resistente. Tesis Doctoral no publicada. Universidad de Granada.

33. Mahamud, J., Tuero, C. y Márquez, S. (2005). Características psicológicas relacionadas con el rendimiento: comparación entre los requerimientos de los entrenadores y la percepción de los deportistas. Revista de Psicología del Deporte, 14(2), 237-251. https://doi.org/10.6018/293641

34. Markovits, A. S. y Hellerman, S. L. (2003). Women's soccer in the United States: yet another American "exceptionalism". Soccer \& Society, 4, 14-29. https://doi.org/10.1080/1466097051233139080 5 . 


\section{Olmedilla et al.}

35. McCormick, A., Meijen, C., Anstiss, P. A. y Jones, H. S. (2017). Self-regulation in endurance sports: theory, research and practice. International Review of Sport and Exercise Psychology.

https://doi.org/10.1080/1750984X.2018.146916 1 .

36. Merino, A., Arraiz, A. y Sabirón, F. (2019). La construcción de la identidad competitiva del niño que práctica fútbol prebenjamín. Revista de Psicología del Deporte, 28(1), 281-289.

37. Moreno-Fernández, I. M., Gómez-Espejo, V., Olmedilla-Caballero, B., Ramos-Pastrana, L. M., Ortega-Toro, E., \& Olmedilla-Zafra, A. (2019). Eficacia de un programa de preparación psicológica en jugadores jóvenes de fútbol. Revista de Psicología Aplicada al Deporte y al Ejercicio Físico, 4, e14, 1-7. https://doi.org/10.5093/rpadef2019e13

38. Morris, T. (2000). Psychological characteristics and talent identification in soccer. Journal of Sports Sciences, 18(9), 715-726. https://doi.org/10.1080/02640410050120096.

39. Murphy, S.M., Fleck, S.J., Dudley, G. y Calister, R. (1990). Psychological and performance concomitants of increase volume training in elite athletes. Journal of Applied Sport Psychology, 2, 34-50. https://doi.org/10.1080/10413209008406419.

40. Olmedilla, A., García-Mas, A. y Ortega, E. (2017). Características psicológicas para el rendimiento deportivo en jóvenes jugadores de fútbol, rugby y baloncesto. Acción Psicológica, 14(1), 7-16. http://dx.doi.org/10.5944/ap.14.1.19249.

41. Olmedilla, A., Moreno-Fernández, I. M., Gómez-Espejo, V., Robles-Palazón, F. J., Verdú, I. y Ortega, E. (2019). Psychological Intervention Program to Control Stress in Youth Soccer Players. Frontiers in Psychology, 10. doi: 10.3389/fpsyg.2019.02260.

42. Olmedilla, A., Ortega, E., Robles-Palazón, F. J., Salom, M. y García-Mas, A. (2018). Healthy practice of female soccer and futsal: identifying sources of stress, anxiety and depression. Sustainability, 10(7), 2268. https://doi.org/10.3390/su10072268.

43. Olmedilla, A., Ruiz-Barquín, R., Ponseti, F. J., Robles-Palazón, F. J. y García-Mas, A. (2019). Competitive psychological disposition and perception of performance in young female soccer players. Frontiers in Psychology, 10. doi: 10.3389/fpsyg.2019.01168.

44. Olmedilla, A., Sánchez-Aldeguer, M. F., Almansa, C. M., Gómez-Espejo, V. y Ortega, E. (2018). Entrenamiento psicológico y mejora de aspectos psicológicos relevantes para el rendimiento deportivo en jugadoras de fútbol. Revista de Psicología Aplicada al Deporte y al Ejercicio Físico, 3, 1-11. https://doi.org/10.5093/rpadef2018a2

45. Olmedilla, A., Torres-Luque, G., GarcíaMas, A., Rubio, V. J., Ducoing, E. y Ortega, E. (2018). Psychological profiling of triathlon and road cycling athletes. Frontiers in Psychology, 9, 825.

https://doi.org/10.3389/fpsyg.2018.00825.

46. Orlick, T. y Partington, J. (1988). Mental links to excellence. The Sport Psychologist, 2, 105-130.

DOI: https://doi.org/10.1123/tsp.2.2.105.

47. Pastrana, J. L., Reigal, R. E., MoralesSánchez, V., Morillo Baro, J. P., Ruiz de Mier, R. J., Alves, J. A. M., \& Hernández-Mendo, A. (2019). Data Mining in the Mixed Methods: Application to the study of the psychological profiles of athletes. Frontiers in Psychology, 10, 2675. https://doi.org/10.3389/fpsyg.2019.02675

48. Pulido-Pedrero, S., de la Vega-Marcos, R. y Fuentes-García, J. P. (2019). Achievement motivation in elite combat athletes: Computerised objective assessment. Revista de Psicologia del Deporte, 29(1), 75-82.

49. Raglin, J. S. (2001). Psychological factors in sport performance. Sports Medicine, 31(12), 875-890. https://doi.org/10.2165/00007256200131120-00004.

50. Reigal, R. E., Delgado, J., López, R. y Hernández-Mendo, A. (2018). Perfil psicológico deportivo y ansiedad estado competitiva en triatletas. Revista de Psicología del Deporte, 


\section{Diferencias psicológicas de género en fútbol juvenil}

27(2), 0125-132. https://doi.org/10.4321/s1578$\underline{84232015000200011}$

51. Stolen, T., Chamari, K., Castagna, C. y Wisloff, U. (2005). Physiology of soccer: an update. Sports Medicine, 35, 501-536. https://doi.org/10.2165/00007256-20053506000004

52. Swann, C., Crust, L., Jackman, P., Vella, S. A., Allen, M. S., and Keegan, R. (2017). Psychological states underlying excellent performance in sport: toward an integrated model of flow and clutch states. Journal of Applied Sport Psychology, 29, 375-401. https://doi.org/10.1080/10413200.2016.127265 $\underline{0}$.

53. Torrebadella-Flix, X. (2016). Fútbol en femenino. Notas para la construcción de una historia social del deporte femenino en España, 1900-1936. Investigaciones Feministas, 7(1), 313-334.

http://dx.doi.org/10.5209/rev_INFE.2016.v7.n1. 52710.

54. Uría, J. (2008). Imágenes de la masculinidad. El futbol español en los años veinte. Ayer, 72, 121-155. https://www.jstor.org/stable/41325993.

55. Vallarino, V. T., García, C. R. y Zecchini, V. Á. (2020). Evaluación e intervención psicológica en jugadoras de hockey sobre hierba femenino. Cuadernos de Psicología del Deporte, 20(1), 62-74. https://doi.org/10.6018/cpd.406651

56. Weinberg, R. S. y Gould, D. (2018). Foundations of sport and exercise psychology, 7E. Human Kinetics.

57. Williams, J. M. y Krane, V. (1998). Psychological characteristics of peak performance. In J.M. Williams (Ed.), Applied sport psychology: Personal growth to peak performance, (3nd ed.), pp. 158-170. Mountain View, CA: Mayfield. 